Reche-Soto, P.; Cardona, D.; Díaz, A.; Gómez-Carmona, C.; Pino-Ortega, J. (2020) ACELT and PLAYER LOAD: Two Variables to Quantify Neuromuscular Load. Revista Internacional de Medicina y Ciencias de la Actividad Física y el Deporte vol. 20 (77) pp. 167-183 Http://cdeporte.rediris.es/revista/revista77/artacelt1110.htm

DOI: $10.15366 /$ rimcafd2020.77.011

\title{
ORIGINAL
}

\section{ACELT Y PLAYER LOAD: DOS VARIABLES PARA LA CUANTIFICACIÓN DE LA CARGA NEUROMUSCULAR}

\section{ACELT AND PLAYER LOAD: TWO VARIABLES TO QUANTIFY NEUROMUSCULAR LOAD}

\author{
Reche-Soto, P.'; Cardona, D. ${ }^{2}$; Díaz, A. ${ }^{3}$; Gómez-Carmona, C. ${ }^{4}$; Pino-Ortega, \\ J. ${ }^{5}$ \\ ${ }^{1}$ Doctorando en Ciencias del Deporte. Facultad de Ciencias de la Actividad Física y del Deporte, \\ Universidad de Murcia (España) pedrorechesoto@gmail.com \\ 2 Doctor en Ciencias del Deporte. Profesor en Facultad de Educación Física, Recreación y \\ Deporte, Politécnico Colombiano Jaime Isaza Cadavid (Colombia) donaldpf@gmail.com \\ ${ }^{3}$ Grupo de Investigación Ingesport. Catedrático y Profesor Titular en Facultad de Ciencias de la \\ Actividad Física y del Deporte, Universidad de Murcia (España) ardiaz@um.es \\ ${ }^{4}$ Grupo de Optimización del Entrenamiento y el Rendimiento Deportivo (GOERD). Doctorando \\ en Ciencias del Deporte. Facultad de Ciencias de la Actividad Física y del Deporte, Universidad \\ de Extremadura (España) cdgomezcarmona@unex.es \\ ${ }^{5}$ Departamento de Actividad Física y Deporte. Doctor y Profesor Titular en Facultad de Ciencias \\ de la Actividad Física y del Deporte, Universidad de Murcia (España) josepinoortega@um.es
}

\section{FINANCIACIÓN}

El autor Carlos D. Gómez Carmona es beneficiario de una beca del Ministerio de Educación, Cultura y Deporte (FPU17/00407). Esta investigación no ha recibido financiación de ningún agente público, privado o sin ánimo de lucro.

\section{CONFLICTO DE INTERESES}

El último autor de este artículo es asesor del área de Ciencias del Deporte en la empresa encargada del desarrollo del dispositivo inercial utilizado. Para garantizar la objetividad de los resultados, este autor no ha contribuido en el análisis de datos ni en la sección de resultados, pero si ha contribuido significativamente en otras partes del manuscrito. Los autores certificamos que este trabajo no ha sido publicado ni está en vías de consideración para su publicación en otra revista, aceptando las normas de publicación de la Revista.

Código UNESCO / UNESCO code: 5899 Otras especialidades pedagógicas (Educación Física y Deporte).

Clasificación Consejo de Europa / Council of Europe Classification: 17. Otras (Rendimiento deportivo) / Other (sport performance). 
Recibido 17 de marzo de 2018 Received March 17, 2018
Aceptado 15 de enero de 2019 Accepted January 15, 2019

\section{RESUMEN}

Los objetivos de esta investigación son: (i) describir el comportamiento de las variables Player Load y AcelT y (ii) cuantificar la carga neuromuscular en diferentes puntos anatómicos (espalda, centro de masas, rodilla y tobillo) durante un test incremental en rampa en tapiz rodante. Veintitrés jugadores semiprofesionales de fútbol varones participaron voluntariamente en este estudio (edad: $22,56 \pm 4,8$ años; masa corporal: $75,5 \pm 5,5 \mathrm{~kg}$; altura: $1,79 \pm 0,5 \mathrm{~m}$ ). Ambas variables se registraron empleando 4 dispositivos inerciales WIMU $\mathrm{PRO}^{\mathrm{TM}}$. Los principales resultados indican que: (1) existe una correlación casi perfecta entre ambas variables $(r=0,931)$, (2) los mayores valores en ambas variables se han encontrado en la rodilla $(P L=8,01 \pm 2,76$; AcelT $=2,70 \pm 0,50)$ y el tobillo ( $P L=7,85 \pm 2,27$; AcelT $=2,87 \pm 0,49)$ y (3) existe una amplia variabilidad intersujeto. En conclusión, Player Load y AcelT son dos indicadores válidos para el análisis y cuantificación de las demandas neuromusculares.

PALABRAS CLAVE: acelerometría, fútbol, rendimiento, AcelT, Player Load.

\section{ABSTRACT}

The objectives of this study were: (i) describe the dynamics of Player Load and AcelT and (ii) analyze the neuromuscular load in different anatomical locations (scapulae, center of mass, knee and ankle) in an incremental test in treadmill. Twenty-three semiprofessional male football players participated voluntarily in this research (age: $22.56 \pm 4.8$ years; body mass: $75.5 \pm 5.5 \mathrm{~kg}$; height: $1.79 \pm 0.5$ m). Four WIMUPRO ${ }^{\mathrm{TM}}$ inertial devices were utilized for recording both variables. The main results indicated that: (1) exists a nearly perfect relation between both variables $(r>0.931)$, (2) the highest values were in knee $\left(P L_{R T}=8.01 \pm 2.76\right.$; AcelT $=2.70 \pm .50)$ and in ankle $(P L=7.85 \pm 2.27$; AcelT $=2.87 \pm .49)$ and $(3)$ a great variability was found between athletes. In conclusion, Player Load and AcelT are two valid variables to analyze and quantify neuromuscular demands.

KEY WORDS: accelerometry, football, performance, AcelT, Player Load.

\section{INTRODUCCIÓN}

Dentro de los deportes colectivos en exterior, el fútbol es uno de los deportes con mayor popularidad, tanto en número de practicantes como en número de aficionados. Este aspecto ha provocado que dicha modalidad deportiva sea una de las más estudiadas en la literatura científica (Sánchez, Yagüe, Fernández y Petisco, 2014). El problema actual es, que la gran complejidad de este deporte, no ha permitido que las investigaciones realizadas hayan reducido por completo la incertidumbre alrededor de las estrategias de entrenamiento para la mejora del rendimiento (Aguiar, Botelho, Lago, Maças y Sampaio, 2012). 
En cuanto a las demandas físicas y fisiológicas, el fútbol es considerado un deporte de equipo con esfuerzos intermitentes de alta intensidad (McMillan, Helgerud, Macdonal y Hoff, 2005), en el que se realizan una gran cantidad de sprints cortos, aceleraciones rápidas, desaceleraciones, giros, saltos, golpeos y tackles, siendo en muchos casos las recuperaciones entre acciones de forma incompleta (Arnason et al, 2004). Todas estas acciones técnico-tácticas dinámicas e impredecibles, que varían en duración e intensidad (Bloomfield, Polman y O'Donoghue, 2007), constituyen la carga interna y externa total que los jugadores experimentan (Akubat, Barrett y Abt, 2014) y suponen un desafío energético para ellos (Stølen, Chamari, Castagna y Wisløff, 2005). La cuantificación precisa y objetiva de las actividades que realizan los jugadores es fundamental para comprender las exigencias físicas del fútbol (Bradley, Di Mascio, Peart y Olsen, 2010; Dwyer y Gabbett, 2012; Johnston et al., 2012), ya que se han registrado hasta 1350 acciones diferentes realizadas por los jugadores (Mohr, Krustrup y Bangsbo, 2003).

Para mejorar los métodos de preparación es necesario ampliar el conocimiento científico. Por ello, se requiere tener un conocimiento objetivo de las características del juego en situaciones de competición (Carling, Williams y Reilly, 2006). Esta posibilidad es, en gran parte, por el desarrollo tecnológico y por la autorización de la FIFA (Federación Internacional de Fútbol Asociado), para el uso en situaciones de competición de dispositivos electrónicos (FIFA, 2018). Estas investigaciones están aportando nuevas evidencias en el estudio de las demandas físicas y tácticas (Castellano y Casamichana, 2014). Disponer de las exigencias de la competición permite orientar de una forma más eficaz las cargas de entrenamiento (Grehaigne, Godbout, y Zerai, 2011) y el diseño de las tareas de entrenamiento tanto a nivel físico (Gómez-Carmona, Gamonales, PinoOrtega e Ibáñez, 2018) como táctico (Reche-Soto, Cardona, Díaz, GómezCarmona y Pino-Ortega, 2019a).

La monitorización de la carga en jugadores de deportes de equipo es una práctica común tanto en entrenamientos como en competición (Rogalski, Dawson, Heasman y Gabbett, 2013). El registro de los parámetros es empleado por los profesionales del deporte con la finalidad de proporcionar una explicación a los posibles cambios de rendimiento del deportista o la de intentar reducir el riesgo de lesión, enfermedad o sobrecarga de entrenamiento (Halson, 2014). El carácter heterogéneo de la respuesta de cada deportista a dicha carga de entrenamiento, hace fundamental su análisis individualizado (Brink, Nederhof, Visscher, Schmikli y Lemmink, 2010; Paulson, Mason, Rhodes y Goosey-Tolfrey, 2015).

Atendiendo a la naturaleza de la carga de trabajo, se clasifica en carga interna y externa, tanto en el ámbito deportivo como científico (Bartlett, O'Connor, Pitchford, Torres-Ronda y Robertson, 2017; Costa et al., 2013). La carga interna, hace referencia al estrés psicológico y fisiológico consecuencia del entrenamiento-competición, mientras que la carga externa abarca el trabajo realizado por el atleta en términos de distancia, velocidad o aceleración (Lambert y Borresen, 2010; McLaren, Weston, Smith, Cramb y Portas, 2016; Paulson et 
al., 2015). En deportes colectivos, existe mayor dificultad en evaluar la carga interna (Borresen y Lambert, 2009), por lo que ha provocado que la cuantificación de la carga externa haya experimentado un mayor desarrollo en los últimos años.

Los acelerómetros se propusieron en la década de 1950 (Culhane, O'Connor, Lyons, y Lyons 2005), y han evolucionado tecnológicamente para ofrecer una calidad y fiabilidad suficiente con un bajo coste de producción que permiten evaluar cuantitativamente de forma portátil el movimiento humano (Lemoyne, Coroian, Mastroianni y Grundfest, 2008). En el contexto deportivo, cada vez más, se utiliza la concepción multifuncional de los dispositivos inerciales (Inertial measurement units, IMUs) que incluyen diferentes sensores como acelerómetro, giróscopo, magnetómetro, GNSS, etc. (Akenhead y Nassis, 2016; Boyd, Ball y Aughey, 2013; Gabbett, 2015).

Uno de los indicadores de carga más utilizado actualmente, a partir de la señal del acelerómetro, es la variable PlayerLoad ${ }^{\mathrm{TM}}$ (PL) (Barrett et al., 2016; Bradley et al., 2010; Dalen, Jørgen, Gertjan, Havard y Ulrik, 2016; Reche-Soto et al., 2019b) o también definido como Body Load (Gomez-Piriz, Jiménez-Reyes y Ruiz-Ruiz, 2011), en función del dispositivo inercial utilizado. Este indicador es la combinación de las aceleraciones producidas en los tres planos del movimiento corporal, produciendo una estimación de la carga total (Cummins, Orr, O'Connor y West, 2013), cuyo resultado es expresado en unidades arbitrarias (Barrett et al., 2016). El PL ha mostrado ser un indicador fiable y valido (Hollville, Couturier, Guilhem y Rabita, 2016), que tiene una alta correlación con variables fisiológicas como frecuencia cardíaca y el $\mathrm{VO}^{2}{ }_{\max }$ (Barrett et al., 2016), y escalas subjetivas de percepción de esfuerzo (Casamichana, Castellano, Calleja-Gonzalez, San Román y Castagna, 2013). Además, esta variable ha obtenido una alta fiabilidad test-retest inter e intra-dispositivo en actividades cíclicas (Barrett et al., 2016), acíclicas (Boyd et al., 2013) y en tareas multidireccionales (ICC $=0,806-0,949)$ (Barreira et al. 2017).

Por todo lo anterior, los objetivos de este estudio son: (i) describir el comportamiento de las variables de carga neuromuscular Player Load y AcelT en un test incremental en rampa en tapiz rodante y (ii) cuantificar la carga neuromuscular en diferentes puntos anatómicos (espalda, zona lumbar, rodilla y tobillo) durante dicha prueba.

\section{MÉTODO}

\subsection{PARTICIPANTES}

Veintitrés jugadores semiprofesionales de fútbol varones (Tercera División, Grupo XIII) participaron voluntariamente en este estudio (Edad: 22,56 $\pm 4,8$ años; Peso: $75,5 \pm 5,5 \mathrm{~kg}$; Altura: 1,79 $\pm 0,5$ metros). Todos los participantes debían cumplir los siguientes requisitos: (i) tener más de dos años de experiencia en la práctica del fútbol a nivel nacional, (ii) presentar más de un año de experiencia con alta monitorización tanto en entrenamientos como partidos oficiales y (iii) no 
presentar ninguna lesión musculo-esquelética o problemas de salud que impidieran su evaluación.

El estudio obtuvo la aprobación del comité de ética de la Universidad de Murcia antes del inicio (Número de registro 2061/2018), conforme al código de ética de la Asociación Médica Mundial (Declaración de Helsinki, 2013). Los participantes fueron informados previamente de los detalles de la investigación y de sus posibles riesgos y beneficios, por lo que para ello se les pasó un consentimiento informado.

\subsection{VARIABLES}

- Player Load (PL): Medición derivada del acelerómetro de la carga corporal total (Body Load) en sus 3 ejes (vertical, antero-posterior y medio-lateral). Esta variable se ha utilizado para evaluar la carga neuromuscular en diferentes atletas (Gómez-Carmona, Pino-Ortega, Sánchez-Ureña, Ibáñez, y RojasValverde, 2019b; Reche-Soto et al., 2019b). Se representa en unidades arbitrarias (a.u.) y se calcula a partir de la siguiente ecuación a una frecuencia de muestreo de $100 \mathrm{~Hz}$ donde $P L_{n}$ es el player load calculado en el instante actual; $n$ es el instante actual de tiempo; $n$ - 1 es el instante previo de tiempo; $X_{n}, Y_{n}$ y $Z_{n}$ son los valores de "Body Load" en cada eje de movimiento en el instante actual; y $X_{n-1}, Y_{n-1}$ y $Z_{n-1}$ son los valores de "Body Load" en cada eje de movimiento en el instante previo:

$$
\begin{gathered}
P L_{n}=\sqrt{\frac{\left(X_{n}-X_{n-1}\right)^{2}+\left(Y_{n}-Y_{n-1}\right)^{2}+\left(Z_{n}-Z_{n-1}\right)^{2}}{100}} \\
P L_{R T}=\sum_{n=0}^{m} P L_{n} \times 0,01
\end{gathered}
$$

- AcelT: magnitud de aceleración o vector resultante (Waldron, Twist, Highton, Worsfold, y Daniels, 2011) identificada como la suma vectorial de la aceleración total registrada por el acelerómetro producto de la gravedad (eje $\mathrm{y}$ ), cambios en el movimiento horizontal (eje $\mathrm{x}$ ) y fuerzas relacionadas con los movimientos de rotación (eje z) de un segmento corporal u objeto al que el acelerómetro esté fijado (O’Donovan, Kamnik, O’Keeffe y Lyons, 2007; Kunze, Bahle, Lukowicz y Partridge, 2010). Se calcula a partir de la siguiente fórmula:

$$
\text { Resultant vector }(\text { AcelT })=\sqrt{x^{2}+y^{2}+z^{2}}
$$




\subsection{INSTRUMENTOS}

\section{Características antropométricas}

La altura de los sujetos fue medida con un tallímetro de pared durante una inspiración máxima (SECA, Hamburgo, Alemania). El peso corporal de los sujetos se obtuvo mediante un monitor de composición corporal modelo BC-601 (TANITA, Tokio, Japón).

\section{Carga neuromuscular}

Ambas variables se registraron empleando 4 dispositivos inerciales WIMUPRO ${ }^{\text {TM }}$ (RealTrack Systems, Almería, España), los cuales contienen cuatro acelerómetros triaxiales que detectan y miden el movimiento usando un sistema electromecánico con una frecuencia de muestreo desde 10 hasta 1.000 $\mathrm{Hz}$. El rango de detección de movimiento es de $\pm 16 \mathrm{~g}, \pm 16 \mathrm{~g}, \pm 32 \mathrm{~g} \mathrm{y} \pm 400 \mathrm{~g}$. Cada dispositivo tiene su propio microprocesador de $1 \mathrm{GHz}, 8 \mathrm{~GB}$ de memoria interna y salida USB de alta velocidad para registrar, almacenar y descargar los datos. Cada dispositivo tiene una batería interna con una duración superior a 4 horas, un peso de $70 \mathrm{~g}$ y unas dimensiones de $81 \times 45 \times 16 \mathrm{~mm}$. En este estudio, los acelerómetros fueron configurados para registrar las variables de investigación con una frecuencia de muestreo de $1.000 \mathrm{~Hz}$.

Los dispositivos fueron colocados en: (i) espalda (C6, entre las escápulas), (ii) lumbar (L3, a la altura del centro de masas) (McGregor, Armstrong, Yaggie, Parshad y Bollt, 2011), (iii) rodilla (a $5 \mathrm{~cm}$ de la grieta de la rótula en sentido ascendente) y (iv) tobillo (a $5 \mathrm{~cm}$ del maléolo lateral en sentido ascendente). Tanto en rodilla como en tobillo fueron colocados en la parte externa de la pierna derecha en todos los sujetos.

Previamente a su colocación, los dispositivos fueron calibrados y sincronizados. Gracias a este proceso los acelerómetros eliminan las 4 fuentes de error que sufren: error de desplazamiento, error de escalamiento, errores ortogonales y error aleatorio (Wang, Liu y Fan, 2006). El proceso de calibración del dispositivo se realizó siguiendo las recomendaciones del fabricante en el proceso de autoarranque. Para asegurar el correcto funcionamiento se deben cumplir tres aspectos: (a) dejar el dispositivo inmóvil durante 30 segundos, (b) en una superficie plana y (c) sin contacto cercano con dispositivos magnéticos (BastidaCastillo, Gómez-Carmona, Reche, Granero-Gil y Pino-Ortega, 2018). Siguiendo este procedimiento, los acelerómetros de este dispositivo han obtenido muy elevados valores de fiabilidad en tests estáticos y dinámicos en diferentes ubicaciones anatómicas (Gómez-Carmona, Bastida-Castillo, García-Rubio, Ibáñez y Pino-Ortega, 2019a)

En la figura 1 se muestran la ubicación de los dispositivos inerciales utilizados, los cuales fueron colocados de la siguiente forma: (i) espalda, mediante un arnés específico ajustado anatómicamente al sujeto y (ii) L3, tobillo y rodilla, mediante 
una banda extensible que fue unida al sujeto con velcro y finalmente se sellaba su anexión reforzándose con cinta adhesiva.

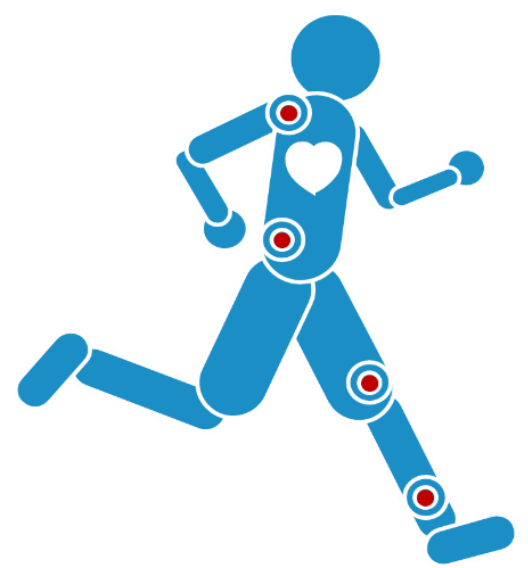

Figura 1. Ubicación de los dispositivos inerciales en los participantes de esta investigación.

\subsection{PROCEDIMIENTO}

El estudio se llevó a cabo durante 2 semanas, con un total de 3 sesiones. En la primera sesión, se registraron las medidas antropométricas y se explicó tanto el protocolo como el objetivo del estudio. La segunda sesión consistió en una familiarización con el test y la realización de carrera a pie con alta monitorización. En la tercera sesión, se realizó el test incremental en rampa en tapiz rodante. La velocidad de inicio fue de $8 \mathrm{~km} / \mathrm{h}$. A partir del inicio, se realizaba un incremento de velocidad continuo de $0,1 \mathrm{~km} / \mathrm{h}$ cada 12 segundos ( $1 \mathrm{~km} / \mathrm{h}$ cada 2 minutos). El test finalizaba cuando el sujeto no conseguía mantener la velocidad.

Todas las sesiones comenzaron a las 9:00 horas y los sujetos debían cumplir los siguientes requisitos: (i) supresión de ingesta de alcohol y cafeína las 24 horas previas a cada una de las sesiones y (ii) no realizar actividad física de alta intensidad las 48 horas previas a la realización del protocolo; para que ninguno de estos factores interfiriese en la investigación (Billat, 2002; Spriet, 2014).

Antes de la realización de cada uno de los protocolos, se realizaba un calentamiento previo estandarizado de 5 minutos de carrera continua al $65 \%$ de la $\mathrm{FC}_{\max }$ que fue monitorizado y controlado en tiempo real mediante el envío de datos de los dispositivos inerciales WIMUPRO ${ }^{\mathrm{TM}}$ (RealTrack Systems, Almería, España) mediante tecnología WiFi a un ordenador el cual disponía del software $S$ PRO $^{\text {TM }}$ (RealTrack Systems, Almería, España) para comprobar el perfecto funcionamiento de los dispositivos (Bastida-Castillo, Gómez-Carmona y PinoOrtega, 2016). Al finalizar el protocolo, se realizaban 5 minutos de carrera continua al $55 \%$ de la FCmax.

\section{ANÁLISIS ESTADÍSTICO}

Para determinar la distribución de los datos se utilizó la prueba Shapiro-Wilk y para la homocedasticidad el test de Levene (Field, 2013). El análisis mostró una distribución normal, por lo que se hicieron pruebas paramétricas. Se realizó un 
primer análisis descriptivo mostrando los resultados mediante medias y desviación estándar. Se realizó una regresión lineal para determinar la relación causa-efecto entre las dos variables de carga neuromuscular Player Load y AcelT en función de la ubicación de los dispositivos inerciales, los sujetos participantes y la velocidad de carrera. Las pruebas estadísticas fueron realizadas mediante el software SPSS 24.0 (SPSS Inc., Chicago IL, USA). La significación estadística se estableció con el valor de $p<0,05$.

\section{RESULTADOS}

En la tabla 1 se realiza el análisis descriptivo de las variables de carga neuromuscular AcelT y Player Load en las diferentes ubicaciones y en función de las diferentes velocidades de carrera a pie. Se encuentran mayores valores en ambas variables cuanto mayor es la velocidad de carrera. Además, también se incrementan los valores conforme la ubicación está más cercana al punto de contacto del pie con el suelo.

Tabla 1. Análisis descriptivo de las variables de carga neuromuscular.

\begin{tabular}{|c|c|c|c|c|c|c|c|c|c|c|c|c|c|c|c|c|}
\hline \multirow{3}{*}{$\begin{array}{l}\text { Velocidad } \\
\qquad(\mathrm{km} / \mathrm{h})\end{array}$} & \multicolumn{8}{|c|}{ Player Load } & \multicolumn{8}{|c|}{ AcelT } \\
\hline & \multicolumn{2}{|c|}{ Espalda } & \multicolumn{2}{|c|}{ L3 } & \multicolumn{2}{|c|}{ Rodilla } & \multicolumn{2}{|c|}{ Tobillo } & \multicolumn{2}{|c|}{ Espalda } & \multicolumn{2}{|c|}{ L3 } & \multicolumn{2}{|c|}{ Rodilla } & \multicolumn{2}{|c|}{ Tobillo } \\
\hline & M & SD & $M$ & SD & $M$ & SD & $M$ & SD & M & SD & $M$ & SD & $M$ & SD & $M$ & SD \\
\hline 8 & 2,26 & 0,35 & 2,37 & 0,42 & 4,90 & 0,63 & 4,81 & 0,82 & 1,19 & 0,04 & 1,30 & 0,06 & 2,05 & 0,09 & 2,13 & 0,10 \\
\hline 8,5 & 2,34 & 0,40 & 2,51 & 0,43 & 5,26 & 0,67 & 5,12 & 0,67 & 1,20 & 0,04 & 1,32 & 0,06 & 2,14 & 0,09 & 2,22 & 0,10 \\
\hline 9 & 2,46 & 0,43 & 2,66 & 0,42 & 5,65 & 0,70 & 5,60 & 0,70 & 1,22 & 0,04 & 1,34 & 0,06 & 2,22 & 0,09 & 2,33 & 0,09 \\
\hline 9,5 & 2,51 & 0,44 & 2,80 & 0,44 & 6,02 & 0,77 & 5,97 & 0,68 & 1,23 & 0,04 & 1,36 & 0,07 & 2,30 & 0,10 & 2,43 & 0,10 \\
\hline 10 & 2,60 & 0,44 & 2,94 & 0,46 & 6,45 & 0,82 & 6,38 & 0,73 & 1,25 & 0,04 & 1,38 & 0,07 & 2,39 & 0,10 & 2,53 & 0,09 \\
\hline 10,5 & 2,70 & 0,46 & 3,08 & 0,47 & 6,80 & 0,90 & 6,74 & 0,75 & 1,26 & 0,04 & 1,40 & 0,08 & 2,46 & 0,11 & 2,62 & 0,10 \\
\hline 11 & 2,80 & 0,45 & 3,22 & 0,46 & 7,44 & 1,55 & 7,08 & 0,83 & 1,27 & 0,04 & 1,42 & 0,08 & 2,59 & 0,22 & 2,72 & 0,10 \\
\hline 11,5 & 2,91 & 0,44 & 3,34 & 0,45 & 7,91 & 1,79 & 7,57 & 0,83 & 1,28 & 0,04 & 1,44 & 0,08 & 2,68 & 0,25 & 2,82 & 0,10 \\
\hline 12 & 3,00 & 0,47 & 3,49 & 0,44 & 8,32 & 1,93 & 7,96 & 0,83 & 1,29 & 0,04 & 1,45 & 0,08 & 2,76 & 0,27 & 2,92 & 0,10 \\
\hline 12,5 & 3,11 & 0,46 & 3,63 & 0,47 & 8,76 & 2,08 & 8,46 & 0,84 & 1,30 & 0,04 & 1,47 & 0,09 & 2,85 & 0,30 & 3,04 & 0,11 \\
\hline 13 & 3,21 & 0,48 & 3,72 & 0,46 & 9,25 & 2,13 & 8,93 & 0,93 & 1,32 & 0,04 & 1,48 & 0,09 & 2,94 & 0,31 & 3,15 & 0,12 \\
\hline 13,5 & 3,30 & 0,46 & 3,87 & 0,47 & 9,78 & 2,29 & 9,46 & 1,04 & 1,33 & 0,04 & 1,50 & 0,08 & 3,05 & 0,36 & 3,25 & 0,13 \\
\hline 14 & 3,47 & 0,51 & 3,93 & 0,43 & 10,53 & 3,00 & 9,99 & 1,06 & 1,34 & 0,04 & 1,51 & 0,08 & 3,22 & 0,53 & 3,36 & 0,12 \\
\hline 14,5 & 3,55 & 0,49 & 4,09 & 0,44 & 11,40 & 4,00 & 10,54 & 1,16 & 1,36 & 0,05 & 1,52 & 0,08 & 3,37 & 0,67 & 3,47 & 0,12 \\
\hline 15 & 3,56 & 0,47 & 4,16 & 0,46 & 10,82 & 1,79 & 11,23 & 1,26 & 1,37 & 0,05 & 1,54 & 0,09 & 3,24 & 0,12 & 3,61 & 0,12 \\
\hline 15,5 & 3,75 & 0,44 & 4,40 & 0,52 & 10,93 & 1,41 & 11,79 & 1,21 & 1,39 & 0,06 & 1,57 & 0,09 & 3,31 & 0,11 & 3,72 & 0,14 \\
\hline 16 & 3,73 & 0,30 & 4,58 & 0,27 & 11,68 & 0,78 & 12,68 & 1,06 & 1,37 & 0,03 & 1,59 & 0,12 & 3,44 & 0,04 & 3,94 & 0,07 \\
\hline Total & 2,94 & 0,63 & 3,37 & 0,75 & 8,01 & 2,76 & 7,85 & 2,27 & 1,28 & 0,07 & 1,43 & 0,11 & 2,70 & 0,50 & 2,87 & 0,49 \\
\hline
\end{tabular}

En la figura 2, se muestra el análisis de regresión entre las variables Player Load y AcelT durante el test incremental en carrera a pie en todos los sujetos analizados. Se encuentran diferentes dinámicas entre la carga del tren superior y la carga del tren inferior a lo largo del espectro de velocidades. Se encuentra una correlación casi perfecta de forma global entre ambas variables de carga neuromuscular $\left(R^{2}=0.931\right)$. 


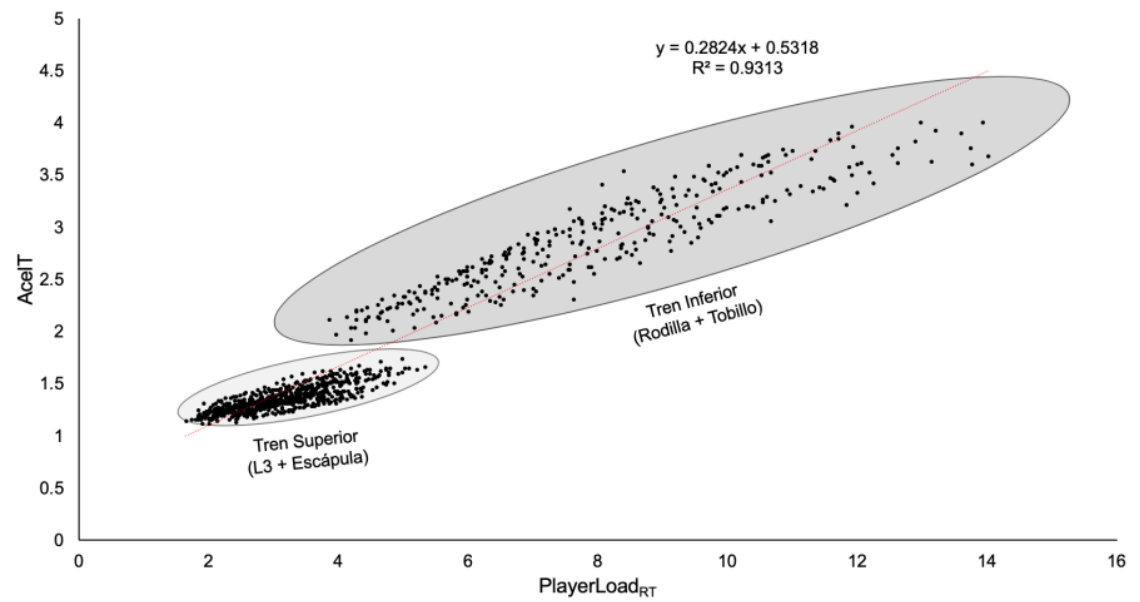

Figura 2. Representación gráfica de la relación entre las variables Player Load y AcelT durante el test incremental progresivo en carrera a pie en tapiz rodante.

En la tabla 2 se muestra la regresión lineal entre las variables de carga neuromuscular AcelT y Player Load en cada uno de los sujetos analizados en las diferentes ubicaciones. Encontramos una variabilidad intersujeto en el análisis por ubicaciones. Además, encontramos las mejores relaciones en las ubicaciones tobillo $\left(R^{2}=0.956\right)$ y rodilla $\left(R^{2}=0.916\right)$ tanto de forma individualizada como global, siendo ambas ubicaciones pertenecientes a la dinámica de carga neuromuscular del tren inferior.

Tabla 2. Regresión lineal entre las variables de carga neuromuscular Player Load y AcelT en las diferentes ubicaciones analizadas en cada uno de los participantes de esta investigación.

\begin{tabular}{ccccc}
\hline Sujetos & Tobillo & Rodilla & L3 & Espalda \\
\hline 1 & 0,997 & 0,958 & 0,998 & 0,973 \\
2 & 0,982 & 0,996 & 0,980 & 0,970 \\
3 & 0,992 & 0,993 & 0,992 & 0,947 \\
4 & 0,992 & 0,993 & 0,992 & 0,947 \\
5 & 0,990 & 0,998 & 0,980 & 0,941 \\
6 & 0,997 & 0,999 & 0,990 & 0,964 \\
7 & 0,996 & 0,984 & 0,992 & 0,967 \\
8 & 0,993 & 0,990 & 0,973 & 0,989 \\
9 & 0,996 & 0,996 & 0,988 & 0,956 \\
10 & 0,996 & 0,998 & 0,984 & 0,923 \\
11 & 0,987 & 0,996 & 0,954 & 0,976 \\
12 & 0,998 & 0,993 & 0,987 & 0,987 \\
13 & 0,993 & 0,979 & 0,997 & 0,988 \\
14 & 0,998 & 0,999 & 0,997 & 0,986 \\
15 & 0,990 & 0,998 & 0,997 & 0,955 \\
16 & 0,992 & 0,997 & 0,956 & 0,952 \\
17 & 0,995 & 0,988 & 0,956 & 0,990 \\
18 & 0,987 & 0,987 & 0,949 & 0,978 \\
19 & 0,990 & 0,994 & 0,962 & 0,987 \\
20 & 0,989 & 0,998 & 0,956 & 0,952 \\
21 & 0,996 & 0,992 & 0,887 \\
22 & 0,995 & 0,998 & 0,993 & 0,713 \\
23 & 0,995 & 0,996 & 0,998 &
\end{tabular}


Finalmente, en la tabla 3 , se realiza un análisis relacional de las variables de carga neuromuscular Player Load y AcelT en las diferentes ubicaciones en función de la velocidad de carrera. Se encuentran mejores relaciones cuanto las velocidades son más altas en las ubicaciones anteriormente mencionadas, tobillo y rodilla.

Tabla 3. Regresión lineal entre las variables de carga neuromuscular Player Load y AcelT en las diferentes ubicaciones analizadas en función de la velocidad de carrera a pie.

\begin{tabular}{cccccc}
\hline \multirow{2}{*}{ Velocidad } & \multicolumn{5}{c}{ Ubicaciones } \\
& Tobillo & Rodilla & L3 & Espalda & Total \\
\hline 8 & 0,806 & 0,628 & 0,520 & 0,139 & 0,879 \\
8,5 & 0,606 & 0,646 & 0,514 & 0,276 & 0,900 \\
9 & 0,590 & 0,577 & 0,531 & 0,331 & 0,910 \\
9,5 & 0,545 & 0,478 & 0,555 & 0,361 & 0,913 \\
10 & 0,535 & 0,440 & 0,551 & 0,424 & 0,916 \\
10,5 & 0,549 & 0,407 & 0,559 & 0,429 & 0,916 \\
11 & 0,765 & 0,789 & 0,513 & 0,469 & 0,906 \\
11,5 & 0,789 & 0,834 & 0,524 & 0,421 & 0,909 \\
12 & 0,847 & 0,834 & 0,543 & 0,394 & 0,908 \\
12,5 & 0,820 & 0,847 & 0,563 & 0,464 & 0,912 \\
13 & 0,817 & 0,841 & 0,566 & 0,550 & 0,915 \\
13,5 & 0,817 & 0,835 & 0,462 & 0,462 & 0,921 \\
14 & 0,907 & 0,904 & 0,420 & 0,546 & 0,935 \\
14,5 & 0,928 & 0,920 & 0,396 & 0,495 & 0,924 \\
15 & 0,786 & 0,676 & 0,379 & 0,494 & 0,928 \\
15,5 & 0,780 & 0,608 & 0,541 & 0,512 & 0,933 \\
16 & 0,781 & 0,897 & 0,567 & 0,549 & 0,972 \\
\hline
\end{tabular}

\section{DISCUSIÓN}

Los objetivos de este estudio han sido: (i) describir el comportamiento de las variables de carga neuromuscular Player Load y AcelT y (ii) cuantificar dichas demandas en diferentes ubicaciones anatómicas a lo largo del espectro de velocidades durante un test incremental en rampa en tapiz rodante.

En el análisis descriptivo realizado, los mayores valores en las variables Player Load y AcelT se han encontrado en la rodilla $(P L=8,01 \pm 2,76$; AcelT $=2,70 \pm$ $0,50)$ y el tobillo ( $P L=7,85 \pm 2,27$; AcelT $=2,87 \pm 0,49$ ), aumentando los valores en ambas variables conforme aumenta la velocidad de carrera. La contribución de la carga desde planos individuales también puede verse influida por la posición anatómica del acelerómetro. Barrett, Midgley y Lovell (2014) encuentran que los valores de ambas variables en las ubicaciones más cercanas al punto de contacto del pie con el suelo aumentan a media que se incrementa la velocidad. Sin embargo, generalmente se acepta que el centro de masa (zona lumbar, L3) (McGregor et al., 2011) es la ubicación anatómica óptima para la colocación de estos dispositivos (Halsey, Shepard y Wilson, 2011; McGregor, Busa, Yaggie y Bollt, 2009), aunque como en nuestro caso hay excepciones en la literatura (Boyd et al., 2013; Scott, Black, Quinn y Coutts, 2013; Cormack et al., 2013). En concreto, en el estudio realizado por Barrett et al., (2014) el PL registrado fue ligeramente mayor en L3 que en espalda. 
En cuanto al análisis de regresión realizado entre las variables de carga acelerómetrica Player Load y AcelT se obtiene que ambas variables tienen una correlación casi perfecta $(r>0,931)$. Por tanto, ambas variables son válidas para la cuantificación y análisis de las demandas neuromusculares. El PL ha sido aceptado como un indicador válido para interpretar la cantidad de carga externa soportada por un jugador en diferentes modalidades deportivas como fútbol (Barreira et al., 2017; Casamichana et al., 2013; Scott et al., 2013), fútbol australiano (Boyd et al., 2013; Gastin, McLean, Spittle y Breed, 2013; Scott et al., 2013) y carrera lineal (Barrett et al., 2014). Investigaciones anteriores han demostrado relaciones significativas y fuertes entre las medidas de carga neuromuscular y el ratio de lesiones en jugadores de rugby (Gabbett, 2004a; Gabbett 2004b), por lo que ambas variables se posicionan como indicadores del riesgo de lesión en deportes de equipo. El PL también presenta alta relación con respecto al índice Edwards ( $r=0,70)$, percepción subjetiva del esfuerzo (PSE) ( $r$ $=0,74)$ y con el volumen de desplazamientos a través de la variable distancia total recorrida $(r=0,70)$ (Casamichana et al., 2013). Scott et al. (2013) también encontró una relación fuerte entre la variable PL y el índice Edwards $(r=0,80)$. En definitiva, los estudios muestran que el PL es un indicador válido para cuantificar las exigencias en fútbol, siendo aceptable para su uso en competición (CV $=1,9 \%)$ (Boyd et al., 2013).

La regresión lineal entre las variables de carga neuromuscular AcelT y Player Load en cada uno de los sujetos analizados en las diferentes ubicaciones muestra una variabilidad intersujeto en el análisis por ubicaciones. Además, encontramos las mejores relaciones en las ubicaciones tobillo $\left(R^{2}=0,956\right)$ y rodilla $\left(R^{2}=0,916\right)$ tanto de forma individualizada como global, siendo las relaciones más altas a una mayor velocidad de carrera. Los resultados obtenidos están en relación a los encontrados en la investigación realizada por Nedergaard et al. (2017) donde existe una baja correlación entre la aceleración del centro de masas (L3) y el resto de ubicaciones (tibia, rodilla y escápula) concluyendo que no se puede detectar la aceleración del cuerpo completo ubicando un acelerómetro en el centro de masas debido a la complejidad de las acciones deportivas. Por tanto, para conseguir la mayor precisión, la ubicación idónea sería el tobillo/tibia debido a que es el primer punto articular que recibe de forma más directa las fuerzas que el sujeto ejerce contra el suelo durante la carrera a pie obteniendo una alta validez con respecto a la plataforma de fuerzas (Raper et al., 2018), consiguiendo detectar diferencias de impacto en función del tipo de calzado (Sinclair y Sant, 2017).

\section{LIMITACIONES DEL ESTUDIO}

Diferentes limitaciones deben tenerse en cuenta al interpretar los resultados obtenidos en esta investigación. En primer lugar, el número de participantes es reducido $(n=23)$, lo cual puede influir en el poder estadístico de los resultados analizados. En segundo lugar, los participantes analizados eran jugadores varones de fútbol de nivel nacional, por lo que los resultados podrían no extrapolarse a otras poblaciones de estudio. Finalmente, únicamente cuatro dispositivos inerciales a una frecuencia de muestreo específica fueron utilizados 
en la recogida de datos. Tanto los componentes del dispositivo inercial, la calibración de los sensores y la frecuencia de muestreo pueden afectar a los resultados obtenidos. Por lo tanto, el registro de los datos mediante los dispositivos inerciales fue realizado siguiendo las recomendaciones del fabricante.

\section{CONCLUSIONES}

Player Load y el AcelT son dos indicadores válidos para el análisis y cuantificación de las demandas neuromusculares. Por tanto, ambos indicadores pueden utilizarse indistintamente para la cuantificación de la carga neuromuscular en entrenamiento y competición. El miembro inferior soporta mayor carga neuromuscular respecto al miembro superior, por tanto, es necesario analizar su dinámica para adaptar las cargas de entrenamiento y los protocolos de recuperación. Finalmente, se encuentra una amplia variabilidad intersujeto, por lo que se recomienda un análisis individualizado de la carga para una mayor especificidad con el objetivo de mejorar el rendimiento deportivo.

\section{REFERENCIAS BIBLIOGRÁFICAS}

Aguiar, M.; Botelho, G.; Lago, C.; Maças, V., \& Sampaio, J. (2012). A review on the effects of soccer small-sided games. Journal of human kinetics, 33, 103-113. https://doi.org/10.2478/v10078-012-0049-x

Akenhead, R., \& Nassis, G. P. (2016). Training load and player monitoring in high-level football: current practice and perceptions. International journal of sports physiology and performance, 11(5), 587-593. https://doi.org/10.1123/ijspp.2015-0331

Akubat, I., Barrett, S., \& Abt, G. (2014). Integrating the internal and external training loads in soccer. International journal of sports physiology and performance, 9(3), 457-462. https://doi.org/10.1123/ijspp.2012-0347

Arnason, A., Sigurdsson, S. B., Gudmundsson, A., Holme, I., Engebretsen, L., \& Bahr, R. (2004). Physical fitness, injuries, and team performance in soccer. Medicine \& Science in Sports \& Exercise, 36(2), 278-285. https://doi.org/10.1249/01.MSS.0000113478.92945.CA

Barreira, P., Robinson, M. A., Drust, B., Nedergaard, N., Raja Azidin, R. M. F., \& Vanrenterghem, J. (2017). Mechanical Player Load ${ }^{\mathrm{TM}}$ using trunkmounted accelerometry in football: Is it a reliable, task-and player-specific observation?. Journal of Sports Sciences, 35(17), 1674-1681. https://doi.org/10.1080/02640414.2016.1229015

Barrett, S., Midgley, A. W., Towlson, C., Garrett, A., Portas, M., \& Lovell, R. (2016). Within-match playerload ${ }^{\mathrm{TM}}$ patterns during a simulated soccer match: Potential implications for unit positioning and fatigue management. International Journal of Sports Physiology and Performance, 11(1), 135140. https://doi.org/10.1123/ijspp.2014-0582

Barrett, S., Midgley, A., \& Lovell, R. (2014). PlayerLoad ${ }^{\mathrm{TM}}$ : reliability, convergent validity, and influence of unit position during treadmill running. International journal of sports physiology and performance, 9(6), 945-952. https://doi.org/10.1123/ijspp.2013-0418 
Bartlett, J. D., O'Connor, F., Pitchford, N., Torres-Ronda, L., \& Robertson, S. J. (2017). Relationships between internal and external training load in teamsport athletes: evidence for an individualized approach. International Journal of Sports Physiology and Performance, 12(2), 230-234. https://doi.org/10.1123/ijspp.2015-0791

Bastida-Castillo, A., Gómez-Carmona, C. D., y Pino-Ortega, J. (2016). Efectos del Tipo de Recuperación Sobre la Oxigenación Muscular Durante el Ejercicio de Sentadilla. Kronos, 15(2).

Bastida-Castillo, A., Gómez-Carmona, C. D., Reche, P., Granero-Gil, P. y PinoOrtega, J. (2018). Valoración de la estabilidad del tronco mediante un dispositivo inercial. Retos: nuevas tendencias en educación física, deporte y recreación, (33), 199-203.

Billat, V. (2002). Fisiología y metodología del entrenamiento. De la teoría a la práctica (Vol. 24). Editorial Paidotribo: España.

Bloomfield, J., Polman, R., \& O'Donoghue, P. (2007). Physical demands of different positions in FA Premier League soccer. Journal of Sports Science and Medicine, 6(1), 63-70.

Borresen, J., \& Lambert, M. I. (2009). The quantification of training load, the training response and the effect on performance. Sports Medicine, 39(9), 779-795.

Boyd, L. J., Ball, K., \& Aughey, R. J. (2013). Quantifying external load in Australian football matches and training using accelerometers. International journal of sports physiology and performance, 8(1), 44-51. https://doi.org/10.1123/ijspp.8.1.44

Bradley, P. S., Di Mascio, M., Peart, D., Olsen, P., \& Sheldon, B. (2010). Highintensity activity profiles of elite soccer players at different performance levels. The Journal of Strength \& Conditioning Research, 24(9), 23432351. https://doi.org/10.1519/JSC.0b013e3181aeb1b3

Brink, M. S., Nederhof, E., Visscher, C., Schmikli, S. L., \& Lemmink, K. A. (2010). Monitoring load, recovery, and performance in young elite soccer players. The Journal of Strength \& Conditioning Research, 24(3), 597603. https://doi.org/10.1519/JSC.0b013e3181c4d38b

Carling, C., Williams, A. M., \& Reilly, T. (2006). Handbook of soccer match analysis: a systematic approach to improving performance. Journal of Sports Science \& Medicine, 5(1), 171.

Casamichana, D., Castellano, J., Calleja-Gonzalez, J., San Román, J., \& Castagna, C. (2013). Relationship between indicators of training load in soccer players. The Journal of Strength \& Conditioning Research, 27(2), 369-374. https://doi.org/10.1519/JSC.0b013e3182548af1

Castellano, J., y Casamichana, D. (2014). Deporte con dispositivos de posicionamiento global (GPS): aplicaciones y limitaciones. Revista de Psicología del Deporte, 23(2), 355-364.

Cormack, S. J., Mooney, M. G., Morgan, W., \& McGuigan, M. R. (2013). Influence of neuromuscular fatigue on accelerometer load in elite Australian football players. International Journal of Sports Physiology and Performance, 8(4), 373-378. https://doi.org/10.1123/ijspp.8.4.373

Costa, E. C., Vieira, C. M., Moreira, A., Ugrinowitsch, C., Castagna, C., \& Aoki, M. S. (2013). Monitoring external and internal loads of Brazilian soccer 
referees during official matches. Journal of Sports Science \& Medicine, 12(3), 559-564.

Culhane, K. M., O'connor, M., Lyons, D., \& Lyons, G. M. (2005). Accelerometers in rehabilitation medicine for older adults. Age and ageing, 34(6), 556-560. https://doi.org/10.1093/ageing/afi192

Cummins, C., Orr, R., O'Connor, H., \& West, C. (2013). Global positioning systems (GPS) and microtechnology sensors in team sports: a systematic review. Sports Medicine, 43(10), 1025-1042. https://doi.org/10.1007/s40279-013-0069-2

Dalen, T., Jørgen, I., Gertjan, E., Havard, H. G., \& Ulrik, W. (2016). Player Load, Acceleration, and Deceleration During Forty-Five Competitive Matches of Elite Soccer. The Journal of Strength \& Conditioning Research, 30(2), 351-359. https://doi.org/10.1519/JSC.0000000000001063

Dwyer, D. B., \& Gabbett, T. J. (2012). Global positioning system data analysis: velocity ranges and a new definition of sprinting for field sport athletes. The Journal of Strength \& Conditioning Research, 26(3), 818-824. https://doi.org/10.1519/JSC.0b013e3182276555

Field, A. (2013). Discovering statistics using IBM SPSS statistics. Sage: Londres.

FIFA (2017). Regla 4. El equipamiento de los jugadores. In: FIFA, ed., Reglas de Juego 2017-2018, 1st ed. Zurich, p.57.

Gabbett, T. J. (2004a). Influence of training and match intensity on injuries in rugby league. Journal of Sports Sciences, 22, 409- 417. https://doi.org/10.1080/02640410310001641638

Gabbett, T. J. (2004b). Reductions in pre-season training loads reduce injury rates in rugby league players. British journal of sports medicine, 38(6), 743-749. http://dx.doi.org/10.1136/bjsm.2003.008391

Gabbett, T. J. (2015). Relationship between accelerometer load, collisions, and repeated high-intensity effort activity in rugby league players. The Journal of Strength \& Conditioning Research, 29(12), 3424-3431. https://doi.org/10.1519/JSC.0000000000001017

Gastin, P. B., McLean, O., Spittle, M., \& Breed, R. V. (2013). Quantification of tackling demands in professional Australian football using integrated wearable athlete tracking technology. Journal of Science and Medicine in Sport, 16(6), 589-593. https://doi.org/10.1016/j.jsams.2013.01.007

Gómez-Carmona, C. D., Bastida-Castillo, A., García-Rubio, J., Ibáñez, S. J., \& Pino-Ortega, J. (2019a). Static and dynamic reliability of WIMU PRO ${ }^{\mathrm{TM}}$ accelerometers according to anatomical placement. Proceedings of the Institution of Mechanical Engineers, Part P: Journal of Sports Engineering and Technology, 233(2), 238-248. https://doi.org/10.1177/1754337118816922

Gómez-Carmona, C., Gamonales, J., Pino-Ortega, J., \& Ibáñez, S. (2018). Comparative Analysis of Load Profile between Small-Sided Games and Official Matches in Youth Soccer Players. Sports, 6, 173. https://doi.org/10.3390/sports6040173

Gómez-Carmona, C. D., Pino-Ortega, J., Sánchez-Ureña, B., Ibáñez, S. J., \& Rojas-Valverde, D. (2019b). Accelerometry-Based External Load Indicators in Sport: Too Many Options, Same Practical Outcome?. International Journal of Environmental Research and Public 
Health, 16(24), 5101. https://doi.org/10.3390/ijerph16245101

Gomez-Piriz, P. T., Jiménez-Reyes, P., \& Ruiz-Ruiz, C. (2011). Relation between total body load and session rating of perceived exertion in professional soccer players. The Journal of Strength \& Conditioning Research, 25(8), 2100-2103. https://doi.org/10.1519/JSC.0b013e3181fb4587

Grehaigne, J. F., Godbout, P., \& Zerai, Z. (2011). How the "rapport de forces" evolves in a soccer match: the dynamics of collective decisions in a complex system. Revista de Psicología del Deporte, 20(2), 747-765.

Halsey, L. G., Shepard, E. L., \& Wilson, R. P. (2011). Assessing the development and application of the accelerometry technique for estimating energy expenditure. Comparative Biochemistry and Physiology Part A: Molecular \& Integrative Physiology, 158(3), 305-314. https://doi.org/10.1016/i.cbpa.2010.09.002

Halson, S. L. (2014). Monitoring training load to understand fatigue in athletes. Sports Medicine, 44(2), 139-147. https://doi.org/10.1007/s40279-0140253-z

Hollville, E., Couturier, A., Guilhem, G., \& Rabita, G. (2016, May). MinimaxX player load as an index of the center of mass displacement? A validation study. In ISBS-Conference Proceedings Archive (Vol. 33, No. 1).

Johnston, R. J., Watsford, M. L., Pine, M. J., Spurrs, R. W., Murphy, A. J., \& Pruyn, E. C. (2012). The validity and reliability of $5-\mathrm{Hz}$ global positioning system units to measure team sport movement demands. The Journal of Strength \& Conditioning Research, 26(3), 758-765. https://doi.org/10.1519/JSC.0b013e318225f161

Kunze, K., Bahle, G., Lukowicz, P., \& Partridge, K. (2010). Can magnetic field sensors replace gyroscopes in wearable sensing applications?. In Wearable Computers (ISWC), 2010 International Symposium on (pp. 14). IEEE.

Lambert, M. I., \& Borresen, J. (2010). Measuring training load in sports. International Journal of Sports Physiology and Performance, 5(3), 406-411. https://doi.org/10.1123/ijspp.5.3.406

Lemoyne, R., Coroian, C., Mastroianni, T., \& Grundfest, W. (2008). Accelerometers for quantification of gait and movement disorders: a perspective review. Journal of Mechanics in Medicine and Biology, 8(02), 137-152. https://doi.org/10.1142/S0219519408002656

Mallo, J., \& Navarro, E. (2008). Physical load imposed on soccer players during small-sided training games. Journal of Sports Medicine and Physical Fitness, 48(2), 166-171.

McGregor, S. J., Armstrong, W. J., Yaggie, J. A., Bollt, E. M., Parshad, R., Bailey, J. J., ... \& Kelly, S. R. (2011). Lower extremity fatigue increases complexity of postural control during a single-legged stance. Journal of Neuroengineering and Rehabilitation, 8(1), 43.

McGregor, S. J., Busa, M. A., Yaggie, J. A., \& Bollt, E. M. (2009). High resolution MEMS accelerometers to estimate VO2 and compare running mechanics between highly trained inter-collegiate and untrained runners. PLoS One, 4(10), e7355. https://doi.org/10.1371/journal.pone.0007355

McLaren, S. J., Weston, M., Smith, A., Cramb, R., \& Portas, M. D. (2016). Variability of physical performance and player match loads in professional 
rugby union. Journal of Science and Medicine in Sport, 19(6), 493-497. https://doi.org/10.1016/j.jsams.2015.05.010

McMillan, K., Helgerud, J., Macdonald, R., \& Hoff, J. (2005). Physiological adaptations to soccer specific endurance training in professional youth soccer players. British Journal of Sports Medicine, 39(5), 273-277. http://dx.doi.org/10.1136/bjsm.2004.012526

Mohr, M., Krustrup, P., \& Bangsbo, J. (2003). Match performance of highstandard soccer players with special reference to development of fatigue. Journal of Sports Sciences, 21(7), 519-528. https://doi.org/10.1080/0264041031000071182

Nedergaard, N. J., Robinson, M. A., Eusterwiemann, E., Drust, B., Lisboa, P. J., \& Vanrenterghem, J. (2017). The Relationship Between Whole-Body External Loading and Body-Worn Accelerometry During Team-Sport Movements. International Journal of Sports Physiology and Performance, 12(1), 18-26. https://doi.org/10.1123/ijspp.2015-0712

O'Donovan, K. J., Kamnik, R., O’Keeffe, D. T., \& Lyons, G. M. (2007). An inertial and magnetic sensor based technique for joint angle measurement. Journal of Biomechanics, 40(12), 2604-2611. https://doi.org/10.1016/i.jbiomech.2006.12.010

Paulson, T. A., Mason, B., Rhodes, J., \& Goosey-Tolfrey, V. L. (2015). Individualized internal and external training load relationships in elite wheelchair rugby players. Frontiers in Physiology, 6, e388. https://doi.org/10.3389/fphys.2015.00388

Raper, D. P., Witchalls, J., Philips, E. J., Knight, E., Drew, M. K., \& Waddington, G. (2018). Use of a tibial accelerometer to measure ground reaction force in running: A reliability and validity comparison with force plates. Journal of Science and Medicine in Sport, 21(1), 84-88. https://doi.org/10.1016/i.jsams.2017.06.010

Reche-Soto, P., Cardona, D., Díaz, A., Gómez-Carmona, C. D., \& Pino-Ortega, J. (2019a). Tactical Demands of Small-Sided Games in Football: Influence of Tracking Technology. Revista Internacional de Medicina y Ciencias de la Actividad Física y el Deporte, 19(76), 729-744.

Reche-Soto, P., Cardona-Nieto, D., Diaz-Suarez, A., Bastida-Castillo, A., Gomez-Carmona, C., Garcia-Rubio, J., \& Pino-Ortega, J. (2019b). Player Load and Metabolic Power Dynamics as Load Quantifiers in Soccer. Journal of Human Kinetics, 69, 259-269. http://www.doi.org/10.2478/hukin-2018-0072

Rogalski, B., Dawson, B., Heasman, J., \& Gabbett, T. J. (2013). Training and game loads and injury risk in elite Australian footballers. Journal of Science and Medicine in Sport, 16(6), 499-503. https://doi.org/10.1016/j.jsams.2012.12.004

Sánchez-Sánchez, J., Yagüe, J. M., Fernández, R. C., \& Petisco, C. (2014). Efectos de un entrenamiento con juegos reducidos sobre la técnica y la condición física de jóvenes futbolistas. RICYDE. Revista Internacional de Ciencias del Deporte, 10(37), 221-234.

Scott, T. J., Black, C. R., Quinn, J., \& Coutts, A. J. (2013). Validity and reliability of the session-RPE method for quantifying training in Australian football: a comparison of the CR10 and CR100 scales. The Journal of Strength \& 
Conditioning Research, 27(1), 270-276. https://doi.org/10.1519/JSC.0b013e3182541d2e

Sinclair, J., \& Sant, B. (2017). The effects of cross-fit footwear on the kinetics and kinematics of running. Footwear Science, 9(1), 41-48. https://doi.org/10.1080/19424280.2016.1268212

Spriet, L. L. (2014). Exercise and sport performance with low doses of caffeine. Sports medicine, 44(2), 175-184. https://doi.org/10.1007/s40279-0140257-8

Stølen, T., Chamari, K., Castagna, C., \& Wisløff, U. (2005). Physiology of soccer. Sports Medicine, 35(6) 501-536. https://doi.org/10.2165/00007256-200535060-00004

Waldron, M., Twist, C., Highton, J., Worsfold, P., \& Daniels, M. (2011). Movement and physiological match demands of elite rugby league using portable global positioning systems. Journal of Sports Sciences, 29(11), 12231230. https://doi.org/10.1080/02640414.2011.587445

Wang, J., Liu, Y., \& Fan, W. (2006). Design and calibration for a smart inertial measurement unit for autonomous helicopters using MEMS sensors. In Mechatronics and Automation, Proceedings of the 2006 IEEE International Conference (pp. 956-961). IEEE.

Número de citas totales / Total references: 63 (100\%)

Número de citas propias de la revista / Journal's own references: $2(3,17 \%)$

Rev.int.med.cienc.act.fís.deporte - vol. 20 - número 77 - ISSN: 1577-0354 